\title{
13. HOW USEFUL ARE HOSPITAL MORBIDITY DATA FOR MONITORING CONDITIONS OCCURRING IN THE PERINATAL PERIOD?
}

The results of this study were originally published in the Australian and New Zealand Journal of Obstetrics and Gynaecology ${ }^{1}$. This version of the article includes additional and extended tables of results. The results section has been modified accordingly.

\section{Reference}

1. Taylor L, Travis S, Pym M, Olive E, Henderson-Smart DJ. How useful are hospital morbidity data for monitoring conditions occurring in the perinatal period? Aust $N \mathrm{ZJ}$ Obstet Gynaecol 2005; 45:36-41.

\section{Introduction}

The main source of information on perinatal morbidity in Australia is state and territory perinatal data collections. Some jurisdictions collect information on all maternal and neonatal conditions, others collect information on a limited set of conditions. Thus there is currently no published information on the full range of conditions affecting mothers and newborns in Australia. It is also recognised that there is variable under-enumeration of those conditions that are covered by perinatal data collections. ${ }^{1,2,3,4,5}$

Data that are routinely collected by hospitals for administrative purposes are a possible source of information on the full range of medical conditions and obstetric complications that may affect mothers and newborn babies, and could potentially supplement information available through perinatal data collections. All hospitals in Australia contribute to state and territory hospital morbidity data collections, which are primarily used for resourcing and management of health services. Hospital morbidity data collections contain information on the reason for admission, known as the primary diagnosis, and on comorbidities including other medical conditions present at the time of admission and medical complications that arise during hospital stay.

There has been a reluctance to use hospital morbidity data collections for monitoring maternal and perinatal morbidity because of concerns about accuracy of the data. A study examining the accuracy of reporting of eclampsia to both the hospital morbidity data collection and the perinatal data collection in Victoria supports this view. ${ }^{6}$ However there is no published information on the accuracy of the range of conditions reported by hospital morbidity data collections.

The aim of this review was to assess the accuracy and reliability of the NSW Inpatient Statistics Collection (ISC) in identifying perinatal morbidity during the hospital admission at time of birth.

\section{Methods}

The ISC covers demographic and episode related data for every inpatient who is separated from any public, private, and repatriation hospital, private day procedure centre, or public nursing home in NSW. It is maintained by the NSW Department of Health's Information Management and Support Unit. From July 1998, the NSW ISC has contained 21 fields for principal diagnosis and comorbidities, which are coded according to the International Classification of Diseases and Related Health Problems (ICD-10-AM). ${ }^{7}$ Conditions reported to the ISC for a sample of records were compared with information obtained through an audit of the corresponding medical records.

A random sample of 1,000 records was selected from computerised records of the ISC: 500 records of mothers who gave birth during the hospital admission and 500 records of liveborn babies born during the admission. Records were selected from NSW public and private hospitals with 50 or more births reported to the ISC in the financial year 1999-00. In 1999-00, 99.2 per cent of deliveries in NSW occurred in hospitals with 50 or more births.

For mothers, records with codes for vaginal or caesarean section deliveries were initially selected (Australian National Diagnosis-Related Group version 4.1 codes O01A, O01B, O01C, O01D, O02Z, O60A, O60B, O60C, or O60D). Records were excluded for hospitals where there were less than 50 mothers giving birth in the financial year (for logistical reasons), for 2 hospitals that had closed, and where the medical record number was not reported. A random number was assigned to each ISC record using a random number generator in SAS version $8.02 .{ }^{8}$ Records were sorted by ascending number and the first 500 records were selected as the sample. For liveborn babies, records indicating a livebirth (code Z38) were selected. The remainder of the sampling procedure was repeated as for mother records.

All selected hospitals agreed to participate in the audit. Each medical record was reviewed independently by a health information manager and a clinical nurse consultant in midwifery, both of whom are Department of Health employees. These staff then compared their findings and came to an agreement on conditions that should be coded. Coding was carried out according to the Australian National Coding Standards, Volume 1. ${ }^{7}$ Data collection was carried out over a 16 month period between October 2002 and February 2004. Coding of conditions was checked for logical consistency by a second health information manager in consultation with the review team, and any discrepancies were corrected by consensus. Information was entered on to a Microsoft Access 2002 database. Information collected through the audit process is referred to as the validation data.

Analyses were carried out using SAS version 8.02. A list of all coded conditions in the validation data was reviewed by an obstetrician and a neonatologist. Clinically important conditions or conditions that were otherwise considered 
to be potentially useful for monitoring the health of mothers and babies over time were retained. The most common exclusions were: non-specific morbidity codes; symptoms, signs and abnormal clinical and laboratory findings; external causes of injury; and factors influencing the person's health status but which are not current illnesses. In addition, certain conditions were grouped. For example, condition codes for degrees of pre-eclampsia were combined into a single group for pre-eclampsia. Codes for premature rupture of membranes were crossmatched with codes for duration of pregnancy to create separate groups for term- and preterm-premature rupture of membranes.

Records in the ISC and validation datasets were compared. Sensitivity, specificity, positive predictive values (PPV) and negative predictive values (NPV) for single codes and grouped codes were calculated using the validation data as the 'gold standard'. Definitions are shown in Table 148. Percentage agreement and kappa measures of agreement were also calculated. Kappa is a measure of inter-rater reliability that corrects for agreement that could occur by chance. A kappa value of one represents perfect agreement and a value of 0 represents only chance agreement. Values greater than 0.75 may be taken to represent excellent agreement beyond chance, values between 0.40 and 0.75 may be taken to represent fair to good agreement beyond chance and values below 0.40 may be taken to represent poor agreement beyond chance. ${ }^{9}$

Published validation studies of Australian state perinatal data collections were reviewed and measures of accuracy and reliability compared with the results obtained in this review.

As this review is an audit of a data collection and conforms to the standards established by the National Health and Medical Research Council for ethical quality review, ${ }^{10}$ ethics committee approval was not sought.

\section{Results}

Of the 1,000 records sampled, 981 were available for review: 490 mother records ( 98.0 per cent) and 491 baby records (98.2 per cent). In the validation data 64 maternal conditions and 45 neonatal conditions were identified that were considered to be relevant to monitoring the health of mothers and babies. Measures of accuracy and reliability are shown in Tables 149 and 150.

Overall agreement was greater than 93 per cent for all conditions. However, many conditions were uncommon, and much of the agreement is due to conditions being correctly recorded as absent on the ISC. Kappa was at a level of 0.75 or above, indicating excellent agreement beyond chance, for 52 (48 per cent) conditions (maternal: $\mathrm{n}=26,41$ per cent; neonatal: $\mathrm{n}=26,58$ per cent); a level of 0.40 to 0.74 , indicating fair to good agreement beyond chance, for a further 35 (32 per cent) conditions (maternal: $\mathrm{n}=26,41$ per cent; neonatal: $\mathrm{n}=9,20$ per cent) neonatal conditions; and a level of less than 0.40 , indicating poor agreement beyond chance, for 22 (20 per cent) (maternal: $\mathrm{n}=12,19$ per cent; neonatal: $\mathrm{n}=10,22$ per cent).

There was a wide range in the sensitivities of reported conditions, with only 34 (32 per cent) conditions (maternal: $\mathrm{n}=12,19$ per cent; neonatal: $\mathrm{n}=22,49$ per cent) having a sensitivity of 100 per cent, and 51 (47 per cent) conditions (maternal: $n=25,39$ per cent; neonatal: $n=26,58$ per cent) having a sensitivity of 80 per cent or more. Nine maternal conditions and 9 neonatal conditions that were found in the validation data were not reported at all in the ISC (sensitivity 0 per cent). These conditions were all uncommon, with only one or 2 cases in the validation data.

PPVs were generally higher than the sensitivities, with 46 (42 per cent) conditions (maternal: $n=22,34$ per cent; neonatal: $n=26,58$ per cent) having a perfect PPV of 100

\section{TABLE 148}

MEASURES OF ACCURACY FOR REPORTED CONDITIONS

\begin{tabular}{|c|c|c|c|}
\hline ISC data & Present & $\begin{array}{l}\text { idation da } \\
\text { Absent }\end{array}$ & Total \\
\hline Present & a & $b$ & $a+b$ \\
\hline Absent & c & d & $c+d$ \\
\hline Total & $a+c$ & $b+d$ & $a+b+c+d$ \\
\hline Term & Formula & \multicolumn{2}{|c|}{ Definition } \\
\hline Sensitivity & $a /(a+c)$ & \multicolumn{2}{|c|}{$\begin{array}{l}\text { Proportion of those with the condition who are reported on the NSW ISC as } \\
\text { having it }\end{array}$} \\
\hline Specificity & $d /(b+d)$ & \multicolumn{2}{|c|}{$\begin{array}{l}\text { Proportion of those without the condition who are reported on the NSW ISC } \\
\text { as not having it }\end{array}$} \\
\hline Positive predictive value (PPV) & $a /(a+b)$ & \multicolumn{2}{|c|}{$\begin{array}{l}\text { Proportion of those reported to have the condition on the NSW ISC who } \\
\text { have the condition }\end{array}$} \\
\hline Negative predictive value (NPV) & $d /(c+d)$ & \multicolumn{2}{|c|}{$\begin{array}{l}\text { Proportion of those reported not to have the condition on the NSW ISC who } \\
\text { do not have the condition }\end{array}$} \\
\hline
\end{tabular}


per cent, and 74 ( 68 per cent) conditions (maternal: $n=41$, 64 per cent; neonatal: $n=33,73$ per cent having a PPV of 80 per cent or more. Levels of specificity, were generally high, with all but 2 maternal and neonatal conditions having specificity of more than 96 per cent. Similarly, NPVs were generally high.

Some of the more common conditions reported are of particular interest for health monitoring purposes. Gestational diabetes was found to have a sensitivity of 96 per cent and almost perfect specificity. While the specificities for gestational hypertension and pre-eclampsia were high at over 99 per cent, the sensitivities were relatively low at 59 per cent and 50 per cent respectively. If these two groups are combined into a general group of 'pregnancy induced hypertension' then the sensitivity rises to 63 per cent, suggesting some misclassification between these two groups. Only two-thirds of all cases of preterm premature rupture of membranes were correctly reported to the ISC (sensitivity 67 per cent). Placenta praevia was reasonably well reported, with a sensitivity of 88 per cent and a specificity of 100 per cent, while placental abruption was less well reported with a sensitivity of 50 per cent and a specificity of 100 per cent. Post partum haemorrhage was similarly under-reported, with a sensitivity of 59 per cent but a high specificity of 99 per cent.

\section{TABLE 149}

COMPARISON OF REPORTING MATERNAL CONDITIONS IN ISC AND VALIDATION DATA: MEASURES OF ACCURACY AND RELIABILITY

\begin{tabular}{|c|c|c|c|c|c|c|c|c|c|}
\hline $\begin{array}{l}\text { ICD-10-AM } \\
\text { code }\end{array}$ & Description & $\begin{array}{l}\text { ISC } \\
\text { data } \\
\text { No. }\end{array}$ & $\begin{array}{l}\text { Validation } \\
\text { data } \\
\text { No. }\end{array}$ & $\begin{array}{c}\text { Sensitivity } \\
\%\end{array}$ & $\begin{array}{c}\text { Specificity } \\
\%\end{array}$ & $\begin{array}{r}\text { PPV } \\
\%\end{array}$ & $\begin{array}{r}\text { NPV } \\
\%\end{array}$ & $\begin{array}{l}\text { Percentage } \\
\text { agreement } \\
\%\end{array}$ & Kappa \\
\hline A53.9 & Syphilis & 0 & 1 & 0.0 & 100.0 & 0.0 & 99.8 & 99.8 & 0.00 \\
\hline A60.0 & $\begin{array}{l}\text { Herpes virus infection of genitalia } \\
\text { and urogenital tract }\end{array}$ & 1 & 2 & 50.0 & 100.0 & 100.0 & 99.8 & 99.8 & 0.67 \\
\hline B01.9 & Varicella & 2 & 2 & 100.0 & 100.0 & 100.0 & 100.0 & 100.0 & 1.00 \\
\hline B18.2 & Chronic viral hepatitis C & 0 & 1 & 0.0 & 100.0 & 0.0 & 99.8 & 99.8 & 0.00 \\
\hline D25.9 & Leiomyoma & 3 & 2 & 100.0 & 99.8 & 66.7 & 100.0 & 99.8 & 0.80 \\
\hline D50 & Iron deficiency anaemia ${ }^{\dagger}$ & 6 & 7 & 57.1 & 99.6 & 66.7 & 99.4 & 99.0 & 0.61 \\
\hline D56 & Thalassemia $^{\dagger}$ & 4 & 4 & 75.0 & 99.8 & 75.0 & 99.8 & 99.6 & 0.75 \\
\hline D50-D64 & Any anaemia ${ }^{\dagger}$ & 5 & 6 & 33.3 & 99.4 & 40.0 & 99.2 & 98.6 & 0.36 \\
\hline D68.0 & Von Willebrand's disease & 1 & 1 & 100.0 & 100.0 & 100.0 & 100.0 & 100.0 & 1.00 \\
\hline D69 & Platelet disorders $^{\dagger}$ & 2 & 5 & 40.0 & 100.0 & 100.0 & 99.4 & 99.4 & 0.57 \\
\hline E03.9,E89.0 & Hypothyroidism $^{\dagger}$ & 6 & 6 & 100.0 & 100.0 & 100.0 & 100.0 & 100.0 & 1.00 \\
\hline E05 & Thyrotoxicosis $^{\dagger}$ & 0 & 3 & 0.0 & 100.0 & 0.0 & 99.4 & 99.4 & 0.00 \\
\hline E06.3 & Autoimmune thyroiditis & 0 & 1 & 0.0 & 100.0 & 0.0 & 99.8 & 99.8 & 0.00 \\
\hline $\mathrm{F} 11.2$ & $\begin{array}{l}\text { Mental and behavioural disorders } \\
\text { due use of opioids- } \\
\text { dependence syndrome }\end{array}$ & 1 & 1 & 100.0 & 100.0 & 100.0 & 100.0 & 100.0 & 1.00 \\
\hline $\mathrm{F} 12.2$ & $\begin{array}{l}\text { Mental and behavioural disorders } \\
\text { due use of cannabinoids- } \\
\text { dependence syndrome }\end{array}$ & 1 & 1 & 100.0 & 100.0 & 100.0 & 100.0 & 100.0 & 1.00 \\
\hline G40.9 & Epilepsy & 2 & 2 & 100.0 & 100.0 & 100.0 & 100.0 & 100.0 & 1.00 \\
\hline J45.9 & Asthma & 3 & 4 & 50.0 & 99.8 & 66.7 & 99.6 & 99.4 & 0.57 \\
\hline M32.1 & $\begin{array}{l}\text { SLE with organ or } \\
\text { system involvement }\end{array}$ & 1 & 1 & 100.0 & 100.0 & 100.0 & 100.0 & 100.0 & 1.00 \\
\hline N18.90 & Chronic renal failure & 0 & 1 & 0.0 & 100.0 & 0.0 & 99.8 & 99.8 & 0.00 \\
\hline 009.3 & $\begin{array}{l}\text { Duration of pregnancy } \\
20-25 \text { completed weeks }\end{array}$ & 0 & 1 & 0.0 & 100.0 & 0.0 & 99.8 & 99.8 & 0.00 \\
\hline 009.4 & $\begin{array}{l}\text { Duration of pregnancy } \\
26-33 \text { completed weeks }\end{array}$ & 5 & 6 & 66.7 & 99.8 & 80.0 & 99.6 & 99.4 & 0.72 \\
\hline 009.5 & $\begin{array}{l}\text { Duration of pregnancy } \\
34-36 \text { completed weeks }\end{array}$ & 11 & 21 & 52.4 & 100.0 & 100.0 & 97.9 & 98.0 & 0.68 \\
\hline O10 & Pre-existing hypertension ${ }^{\dagger}$ & 7 & 7 & 85.7 & 99.8 & 85.7 & 99.8 & 99.6 & 0.86 \\
\hline 013 & Gestational hypertension & 21 & 29 & 58.6 & 99.1 & 81.0 & 97.4 & 96.7 & 0.66 \\
\hline O14 & Pre-eclampsia $^{\dagger}$ & 12 & 22 & 50.0 & 99.8 & 91.7 & 97.7 & 97.6 & 0.64 \\
\hline O13,014 & Pregnancy induced hypertension ${ }^{\dagger}$ & 33 & 51 & 62.7 & 99.8 & 97.0 & 95.8 & 95.9 & 0.74 \\
\hline O23.4 & Urinary tract infection in pregnancy & 4 & 3 & 33.3 & 99.4 & 25.0 & 99.6 & 99.0 & 0.28 \\
\hline O24.0 & Diabetes in pregnancy & 1 & 2 & 50.0 & 100.0 & 100.0 & 99.8 & 99.8 & 0.67 \\
\hline O24.4 & Gestational diabetes & 22 & 22 & 95.5 & 99.8 & 95.5 & 99.8 & 99.6 & 0.95 \\
\hline O30.0 & Twin pregnancy & 4 & 3 & 100.0 & 99.8 & 75.0 & 100.0 & 99.8 & 0.86 \\
\hline
\end{tabular}


TABLE 149 (continued)

COMPARISON OF REPORTING MATERNAL CONDITIONS IN ISC AND VALIDATION DATA: MEASURES OF ACCURACY AND RELIABILITY

\begin{tabular}{|c|c|c|c|c|c|c|c|c|c|}
\hline $\begin{array}{l}\text { ICD-10-AM } \\
\text { code }\end{array}$ & Description & $\begin{array}{l}\text { ISC } \\
\text { data } \\
\text { No. }\end{array}$ & $\begin{array}{l}\text { Validation } \\
\text { data } \\
\text { No. }\end{array}$ & $\begin{array}{c}\text { Sensitivity } \\
\%\end{array}$ & $\begin{array}{c}\text { Specificity } \\
\%\end{array}$ & $\begin{array}{l}\text { PPV } \\
\%\end{array}$ & $\begin{array}{l}\text { NPV } \\
\%\end{array}$ & $\begin{array}{c}\text { Percentage } \\
\text { agreement } \\
\%\end{array}$ & Kappa \\
\hline O34.2 & $\begin{array}{l}\text { Maternal care due to uterine } \\
\text { scar from previous surgery }\end{array}$ & 40 & 40 & 92.5 & 99.3 & 92.5 & 99.3 & 98.8 & 0.92 \\
\hline O36.0,036.1 & Maternal care for isoimmunisation ${ }^{\dagger}$ & 7 & 8 & 62.5 & 99.6 & 71.4 & 99.4 & 99.0 & 0.66 \\
\hline O41.1 & $\begin{array}{l}\text { Infection of amniotic } \\
\text { sac and membranes }\end{array}$ & 0 & 2 & 0.0 & 100.0 & 0.0 & 99.6 & 99.6 & 0.00 \\
\hline O42.0 & $\begin{array}{l}\text { Premature rupture of membranes: } \\
\text { onset of labour within } 24 \text { hours }\end{array}$ & 21 & 23 & 47.8 & 97.9 & 52.4 & 97.4 & 95.5 & 0.48 \\
\hline O42.1 & $\begin{array}{l}\text { Premature rupture of membranes: } \\
\text { onset of labour after } 24 \text { hours }\end{array}$ & 13 & 19 & 57.9 & 99.6 & 84.6 & 98.3 & 98.0 & 0.68 \\
\hline O42.11 & $\begin{array}{l}\text { Premature rupture of membranes: } \\
\text { onset of labour between } \\
1-7 \text { days later }\end{array}$ & 11 & 13 & 69.2 & 99.6 & 81.8 & 99.2 & 98.8 & 0.74 \\
\hline O42.12 & $\begin{array}{l}\text { Premature rupture of membranes: } \\
\text { onset of labour more than } \\
7 \text { days later }\end{array}$ & 2 & 4 & 25.0 & 99.8 & 50.0 & 99.4 & 99.2 & 0.33 \\
\hline 042.2 & $\begin{array}{l}\text { Premature rupture of membranes: } \\
\text { labour delayed by therapy }\end{array}$ & 0 & 1 & 0.0 & 100.0 & 0.0 & 99.8 & 99.8 & 0.00 \\
\hline 042.9 & $\begin{array}{l}\text { Premature rupture of membranes: } \\
\text { unspecified }\end{array}$ & 2 & 1 & 100.0 & 99.8 & 50.0 & 100.0 & 99.8 & 0.67 \\
\hline \multirow[t]{3}{*}{$\mathrm{O} 42$} & $\begin{array}{l}\text { Any premature rupture } \\
\text { of membranes }{ }^{\dagger}\end{array}$ & 36 & 44 & 61.4 & 98.0 & 75.0 & 96.3 & 94.7 & 0.65 \\
\hline & $\begin{array}{l}\text { Preterm premature rupture } \\
\text { of membranes (PPROM) }\end{array}$ & 6 & 9 & 66.7 & 100.0 & 100.0 & 99.4 & 99.4 & 0.80 \\
\hline & $\begin{array}{l}\text { Term premature rupture } \\
\text { of membranes (TPROM) }\end{array}$ & 30 & 35 & 54.3 & 97.6 & 63.3 & 96.5 & 94.5 & 0.56 \\
\hline O44 & Placenta praevia ${ }^{\dagger}$ & 7 & 8 & 87.5 & 100.0 & 100.0 & 99.8 & 99.8 & 0.93 \\
\hline $\mathrm{O} 45$ & Placental abruption ${ }^{\dagger}$ & 2 & 4 & 50.0 & 100.0 & 100.0 & 99.6 & 99.6 & 0.66 \\
\hline O48 & Prolonged pregnancy & 45 & 47 & 95.7 & 100.0 & 100.0 & 99.6 & 99.6 & 0.98 \\
\hline O64-O66 & Obstructed labour ${ }^{\dagger}$ & 33 & 40 & 75.0 & 99.3 & 90.9 & 97.8 & 97.3 & 0.81 \\
\hline $\mathrm{O} 68$ & $\begin{array}{l}\text { Labour and delivery complicated } \\
\text { by fetal stress (distress) }{ }^{\dagger}\end{array}$ & 76 & 72 & 87.5 & 96.9 & 82.9 & 97.8 & 95.5 & 0.82 \\
\hline 069.1 & $\begin{array}{l}\text { Labour and delivery complicated } \\
\text { by cord around neck, } \\
\text { with compression }\end{array}$ & 13 & 15 & 80.0 & 99.8 & 92.3 & 99.4 & 99.2 & 0.85 \\
\hline 069.4 & $\begin{array}{l}\text { Labour and delivery complicated } \\
\text { by vasa previa }\end{array}$ & 1 & 2 & 50.0 & 100.0 & 100.0 & 99.8 & 99.8 & 0.67 \\
\hline 070.0 & First degree perineal laceration & 69 & 64 & 92.2 & 97.7 & 85.5 & 98.8 & 96.9 & 0.87 \\
\hline 070.1 & Second degree perineal laceration & 91 & 94 & 94.7 & 99.5 & 97.8 & 98.7 & 98.6 & 0.95 \\
\hline 070.2 & Third degree perineal laceration & 10 & 11 & 90.9 & 100.0 & 100.0 & 99.8 & 99.8 & 0.95 \\
\hline 070.3 & Fourth degree perineal laceration & 1 & 1 & 100.0 & 100.0 & 100.0 & 100.0 & 100.0 & 1.00 \\
\hline O70 & Any perineal laceration ${ }^{\dagger}$ & 171 & 170 & 95.3 & 97.2 & 94.7 & 97.5 & 96.5 & 0.92 \\
\hline $\mathrm{O} 72$ & Post partum haemorrhage $^{\dagger}$ & 18 & 29 & 58.6 & 99.8 & 94.4 & 97.5 & 97.3 & 0.71 \\
\hline 075.7 & $\begin{array}{l}\text { Vaginal delivery following } \\
\text { previous caesarean section }\end{array}$ & 4 & 11 & 36.4 & 100.0 & 100.0 & 98.6 & 98.6 & 0.53 \\
\hline \multicolumn{2}{|c|}{ O85,O86.0,086.2 Postpartum infection ${ }^{\dagger}$} & 7 & 12 & 50.0 & 99.8 & 85.7 & 98.8 & 98.6 & 0.62 \\
\hline 090.1 & $\begin{array}{l}\text { Disruption of perineal } \\
\text { obstetric wound }\end{array}$ & 1 & 2 & 50.0 & 100.0 & 100.0 & 99.8 & 99.8 & 0.67 \\
\hline 090.2 & Haematoma of obstetric wound & 2 & 1 & 100.0 & 99.8 & 50.0 & 100.0 & 99.8 & 0.67 \\
\hline Z37.0 & Single live birth $\S$ & 474 & 472 & 99.4 & 72.2 & 98.9 & 81.3 & 98.4 & 0.76 \\
\hline Z37.1 & Single stillbirth $\S$ & 6 & 8 & 75.0 & 100.0 & 100.0 & 99.6 & 99.6 & 0.86 \\
\hline Z37.2 & Twins, both liveborn $\S$ & 5 & 6 & 83.3 & 100.0 & 100.0 & 99.8 & 99.8 & 0.91 \\
\hline Z37.5 & Other multiple births, all liveborn & 0 & 1 & 0.0 & 100.0 & & 99.8 & 99.8 & 0.00 \\
\hline Z72.0 & Tobacco use, current & 57 & 80 & 66.3 & 99.0 & 93.0 & 93.8 & 93.7 & 0.74 \\
\hline \multicolumn{10}{|c|}{$\begin{array}{l}\text { † Conditions for which ICD-10 codes were grouped } \\
\text { † PPROM categorised using any code for premature rupture of membranes (042) with any code for duration of pregnancy less than } 37 \text { weeks (O09.0- } \\
\text { O09.5). Other cases of premature rupture of membranes classified as TPROM. } \\
\S \text { Number of births do not sum to } 490 \text { as } 3 \text { mothers gave birth prior to arrival at hospital. }\end{array}$} \\
\hline
\end{tabular}




\section{TABLE 150}

COMPARISON OF REPORTING OF NEONATAL CONDITIONS IN NSW ISC AND VALIDATION DATA: MEASURES OF ACCURACY AND RELIABILITY

\begin{tabular}{|c|c|c|c|c|c|c|c|c|c|}
\hline $\begin{array}{l}\text { ICD-10-AM } \\
\text { code }\end{array}$ & Description & $\begin{array}{l}\text { ISW ISC } \\
\text { data } \\
\text { No. }\end{array}$ & $\begin{array}{l}\text { Validation } \\
\text { data } \\
\text { No. }\end{array}$ & $\begin{array}{c}\text { Sensitivity } \\
\%\end{array}$ & $\begin{array}{c}\text { Specificity } \\
\%\end{array}$ & $\begin{array}{l}\text { PPV } \\
\%\end{array}$ & $\begin{array}{l}\text { NPV } \\
\%\end{array}$ & $\begin{array}{l}\text { Percentage } \\
\text { agreement } \\
\%\end{array}$ & Kappa \\
\hline P07.2 & $\begin{array}{l}\text { Extreme immaturity } \\
\text { (Less than } 28 \text { completed weeks) }\end{array}$ & 2 & 2 & 100.0 & 100.0 & 100.0 & 100.0 & 100.0 & 1.00 \\
\hline P07.3 & $\begin{array}{l}\text { Other preterm infants ( } 28 \text { completed } \\
\text { weeks to } 37 \text { completed weeks) }\end{array}$ & ed 24 & 31 & 74.2 & 99.8 & 95.8 & 98.3 & 98.2 & 0.83 \\
\hline E84.1 & $\begin{array}{l}\text { Cystic fibrosis with intestinal } \\
\text { manifestations }\end{array}$ & 1 & 1 & 100.0 & 100.0 & 100.0 & 100.0 & 100.0 & 1.00 \\
\hline E87.1 & $\begin{array}{l}\text { Hypo-osmolality and } \\
\text { hyponatraemia }\end{array}$ & 0 & 1 & 0.0 & 100.0 & 0.0 & 99.8 & 99.8 & 0.00 \\
\hline E87.2 & Acidosis & 1 & 1 & 100.0 & 100.0 & 100.0 & 100.0 & 100.0 & 1.00 \\
\hline K21.9 & $\begin{array}{l}\text { Gastro-oesophageal reflux } \\
\text { disease without oesophagitis }\end{array}$ & 1 & 1 & 0.0 & 99.8 & 0.0 & 99.8 & 99.6 & 0.00 \\
\hline K42.9 & $\begin{array}{l}\text { Umbilical hernia without } \\
\text { obstruction or gangrene }\end{array}$ & 1 & 1 & 100.0 & 100.0 & 100.0 & 100.0 & 100.0 & 1.00 \\
\hline P05.0,P05.1 & Small for gestational age & 0 & 0 & 0.0 & 100.0 & 0.0 & 100.0 & 100.0 & 0.00 \\
\hline P07.0 & $\begin{array}{l}\text { Extremely low birth weight } \\
\text { (999 gms or less) }\end{array}$ & 1 & 1 & 100.0 & 100.0 & 100.0 & 100.0 & 100.0 & 1.00 \\
\hline P07.1 & $\begin{array}{l}\text { Other low birth weight } \\
(1,000-2,499 \text { gms })\end{array}$ & 12 & 11 & 100.0 & 99.8 & 91.7 & 100.0 & 99.8 & 0.96 \\
\hline $\begin{array}{l}\text { P11.3,P13.1, } \\
\text { P13.3,P13.4 }\end{array}$ & Birth injuries $^{\dagger}$ & 0 & 0 & 0.0 & 100.0 & 0.0 & 100.0 & 100.0 & 0.00 \\
\hline P21.0 & $\begin{array}{l}\text { Severe birth asphyxia } \\
\text { (Apgar score } 0-3 \text { at } 1-\text { minute) }\end{array}$ & 1 & 2 & 50.0 & 100.0 & 100.0 & 99.8 & 99.8 & 0.67 \\
\hline P21.1 & $\begin{array}{l}\text { Mild and moderate birth asphyxia } \\
\text { (Apgar score } 4-7 \text { at one minute) }\end{array}$ & 3 & 1 & 100.0 & 99.6 & 33.3 & 100.0 & 99.6 & 0.50 \\
\hline P22.0 & $\begin{array}{l}\text { Respiratory distress of newborn } \\
\text { (hyaline membrane disease) }\end{array}$ & 7 & 14 & 50.0 & 100.0 & 100.0 & 98.6 & 98.6 & 0.66 \\
\hline P22.1 & Transient tachypnoea of newborn & 9 & 11 & 63.6 & 99.6 & 77.8 & 99.2 & 98.8 & 0.69 \\
\hline P24.0 & Neonatal aspiration of meconium & 1 & 4 & 25.0 & 100.0 & 100.0 & 99.4 & 99.4 & 0.40 \\
\hline P24.2 & Neonatal aspiration of blood & 1 & 1 & 100.0 & 100.0 & 100.0 & 100.0 & 100.0 & 1.00 \\
\hline P28.2 & Cyanotic attacks of newborn & 1 & 1 & 100.0 & 100.0 & 100.0 & 100.0 & 100.0 & 1.00 \\
\hline P28.3 & Primary sleep apnoea of newborn & 1 & 1 & 100.0 & 100.0 & 100.0 & 100.0 & 100.0 & 1.00 \\
\hline P29.2 & Neonatal hypertension & 1 & 1 & 100.0 & 100.0 & 100.0 & 100.0 & 100.0 & 1.00 \\
\hline P36.0 & $\begin{array}{l}\text { Sepsis of newborn due } \\
\text { to streptococcus, group B }\end{array}$ & 0 & 1 & 0.0 & 100.0 & 0.0 & 99.8 & 99.8 & 0.00 \\
\hline P52.0 & $\begin{array}{l}\text { Intraventricular (nontraumatic) } \\
\text { haemorrhage, grade } 1 \text {, } \\
\text { of fetus and newborn }\end{array}$ & 2 & 2 & 100.0 & 100.0 & 100.0 & 100.0 & 100.0 & 1.00 \\
\hline P52.1 & $\begin{array}{l}\text { Intraventricular (nontraumatic) } \\
\text { haemorrhage, grade } 2 \text {, } \\
\text { of fetus and newborn }\end{array}$ & 1 & 1 & 100.0 & 100.0 & 100.0 & 100.0 & 100.0 & 1.00 \\
\hline P52.5 & $\begin{array}{l}\text { Subarachnoid (nontraumatic) } \\
\text { haemorrhage of } \\
\text { fetus and newborn }\end{array}$ & 1 & 1 & 100.0 & 100.0 & 100.0 & 100.0 & 100.0 & 1.00 \\
\hline P55 & $\begin{array}{l}\text { Haemolytic disease of } \\
\text { fetus and newborn* }\end{array}$ & 3 & 5 & 60.0 & 100.0 & 100.0 & 99.6 & 99.6 & 0.75 \\
\hline P59.0-P59.9 & Neonatal jaundice $^{\dagger}$ & 22 & 25 & 80.0 & 99.6 & 90.9 & 98.9 & 98.6 & 0.84 \\
\hline P70.0-P70.4 & Neonatal hypoglycaemia ${ }^{\dagger}$ & 18 & 20 & 85.0 & 99.8 & 94.4 & 99.4 & 99.2 & 0.89 \\
\hline P75 & Meconium ileus & 1 & 1 & 100.0 & 100.0 & 100.0 & 100.0 & 100.0 & 1.00 \\
\hline P90 & Convulsions of newborn & 1 & 1 & 0.0 & 99.8 & 0.0 & 99.8 & 99.6 & 0.00 \\
\hline P92.0-P92.9 & Feeding problems of newborn ${ }^{\dagger}$ & 20 & 35 & 48.6 & 99.3 & 85.0 & 96.2 & 95.7 & 0.60 \\
\hline P96.1 & $\begin{array}{l}\text { Neonatal withdrawal symptoms } \\
\text { from maternal use } \\
\text { of drugs of addiction }\end{array}$ & 2 & 2 & 100.0 & 100.0 & 100.0 & 100.0 & 100.0 & 1.00 \\
\hline Q03.9 & Congenital hydrocephalus & 1 & 1 & 100.0 & 100.0 & 100.0 & 100.0 & 100.0 & 1.00 \\
\hline Q21.0 & Ventricular septal defect & 1 & 1 & 100.0 & 100.0 & 100.0 & 100.0 & 100.0 & 1.00 \\
\hline Q33.9 & $\begin{array}{l}\text { Congenital malformation of } \\
\text { lung, unspecified }\end{array}$ & 0 & 1 & 0.0 & 100.0 & 0.0 & 99.8 & 99.8 & 0.00 \\
\hline Q53.1 & Undescended testicle, unilateral & 2 & 4 & 50.0 & 100.0 & 100.0 & 99.6 & 99.6 & 0.66 \\
\hline Q53.2 & Undescended testicle, bilateral & 1 & 2 & 50.0 & 100.0 & 100.0 & 99.8 & 99.8 & 0.67 \\
\hline
\end{tabular}


TABLE 150 (continued)

COMPARISON OF REPORTING OF NEONATAL CONDITIONS IN ISC AND VALIDATION DATA:

MEASURES OF ACCURACY AND RELIABILITY

\begin{tabular}{|c|c|c|c|c|c|c|c|c|c|}
\hline $\begin{array}{l}\text { ICD-10-AM } \\
\text { code }\end{array}$ & Description & $\begin{array}{l}\text { ISC } \\
\text { data } \\
\text { No. }\end{array}$ & $\begin{array}{c}\text { Validation } \\
\text { data } \\
\text { No. }\end{array}$ & $\begin{array}{c}\text { Sensitivity } \\
\%\end{array}$ & $\begin{array}{c}\text { Specificity } \\
\%\end{array}$ & $\begin{array}{r}\text { PPV } \\
\%\end{array}$ & $\begin{array}{c}\text { NPV } \\
\%\end{array}$ & $\begin{array}{c}\text { Percentage } \\
\text { agreement } \\
\%\end{array}$ & Kappa \\
\hline Q54.9 & Hypospadias & 1 & 1 & 100.0 & 100.0 & 100.0 & 100.0 & 100.0 & 1.00 \\
\hline Q65.0 & $\begin{array}{l}\text { Congenital dislocation of hip, } \\
\text { unilateral }\end{array}$ & 1 & 1 & 100.0 & 100.0 & 100.0 & 100.0 & 100.0 & 1.00 \\
\hline Q66.0 & Talipes equinovarus & 2 & 2 & 50.0 & 99.8 & 50.0 & 99.8 & 99.6 & 0.50 \\
\hline Q66.1 & Talipes calcaneovarus & 0 & 1 & 0.0 & 100.0 & 0.0 & 99.8 & 99.8 & 0.00 \\
\hline Q66.2 & Metatarsus varus & 1 & 1 & 100.0 & 100.0 & 100.0 & 100.0 & 100.0 & 1.00 \\
\hline Q66.4 & Talipes calcaneovalgus & 1 & 1 & 100.0 & 100.0 & 100.0 & 100.0 & 100.0 & 1.00 \\
\hline Z38.0 & Singleton, born in hospital & 472 & 471 & 99.8 & 90.0 & 99.6 & 94.7 & 99.4 & 0.92 \\
\hline Z38.2 & $\begin{array}{l}\text { Singleton, unspecified } \\
\text { as to place of birth }\end{array}$ & 0 & 1 & 0.0 & 100.0 & 0.0 & 99.8 & 99.8 & 0.00 \\
\hline Z38.3 & Twin, born in hospital & 19 & 19 & 94.7 & 99.8 & 94.7 & 99.8 & 99.6 & 0.95 \\
\hline
\end{tabular}

\section{TABLE 151}

COMPARISON OF NSW ISC AND OTHER STATE PERINATAL DATA COLLECTIONS FOR SELECTED CONDITIONS

\begin{tabular}{|c|c|c|c|c|c|c|c|}
\hline Condition & Data collection ${ }^{\dagger}$ & $\begin{array}{c}\text { Sensitivity } \\
\%\end{array}$ & $\begin{array}{c}\text { Specificity } \\
\%\end{array}$ & $\begin{array}{c}\text { PPV } \\
\%\end{array}$ & $\begin{array}{r}\text { NPV } \\
\%\end{array}$ & $\begin{array}{c}\text { Percentage } \\
\%\end{array}$ & Kappa \\
\hline \multirow[t]{2}{*}{ Premature rupture of membranes } & NSW ISC & 61.4 & 98.0 & 75.0 & 96.3 & 94.7 & 0.65 \\
\hline & WA perinatal data collection & 75.0 & 98.6 & 63.2 & 99.2 & 97.8 & - \\
\hline \multirow[t]{4}{*}{ Pre-existing hypertension } & NSW ISC & 85.7 & 99.8 & 85.7 & 99.8 & 99.6 & 0.86 \\
\hline & Victorian perinatal data collection & 71.4 & - & - & - & - & - \\
\hline & SA perinatal data collection & - & - & - & - & 99.3 & 0.72 \\
\hline & NSW perinatal data collection & 62.5 & 99.8 & - & - & 99.6 & 0.59 \\
\hline \multirow[t]{4}{*}{ Pre-eclampsia } & NSW ISC & 50.0 & 99.8 & 91.7 & 97.7 & 97.6 & 0.64 \\
\hline & Victorian perinatal data collection & 87.0 & - & - & - & - & - \\
\hline & WA perinatal data collection & 65.6 & 98.1 & 70.0 & 97.7 & 96.1 & - \\
\hline & NSW perinatal data collection & 66.7 & 99.3 & - & - & 96.4 & 0.75 \\
\hline \multirow[t]{3}{*}{ Pregnancy induced hypertension } & NSW ISC & 62.7 & 99.8 & 97.0 & 95.8 & 95.9 & 0.74 \\
\hline & Victorian perinatal data collection & 60.0 & - & - & - & - & - \\
\hline & SA perinatal data collection & - & - & - & - & 98.3 & 0.87 \\
\hline \multirow[t]{3}{*}{ Gestational diabetes } & NSW ISC & 95.5 & 99.8 & 95.5 & 99.8 & 99.6 & 0.95 \\
\hline & WA perinatal data collection & 100.0 & 99.2 & 69.2 & 100.0 & 99.2 & - \\
\hline & NSW perinatal data collection & 86.7 & 99.6 & - & - & 99.1 & 0.87 \\
\hline \multirow[t]{4}{*}{ Postpartum haemorrhage } & NSW ISC & 58.6 & 99.8 & 94.4 & 97.5 & 97.3 & 0.71 \\
\hline & Victorian perinatal data collection & 100.0 & - & - & - & - & - \\
\hline & SA perinatal data collection & - & - & - & - & 96.5 & 0.68 \\
\hline & WA perinatal data collection & 80.0 & 99.4 & 88.9 & 98.8 & 98.2 & - \\
\hline \multirow[t]{3}{*}{ Fetal distress } & NSW ISC & 87.5 & 96.9 & 82.9 & 97.8 & 95.5 & 0.82 \\
\hline & SA perinatal data collection & - & - & - & - & 95.3 & 0.79 \\
\hline & WA perinatal data collection & 90.5 & 93.3 & 65.5 & 98.6 & 92.9 & - \\
\hline \multirow[t]{2}{*}{ Third degree perineal laceration } & NSW ISC & 90.9 & 100.0 & 100.0 & 99.8 & 99.8 & 0.95 \\
\hline & SA perinatal data collection & - & - & - & - & 100.0 & 1.00 \\
\hline \multicolumn{8}{|c|}{$\begin{array}{l}\text { † Conditions presented where } 10 \text { or more cases reported from medical records in the NSW ISC and comparative published information available from } \\
\text { perinatal data collections. } \\
\text { Sources: Publications listed in the references as follows: SA perinatal data collection }{ }^{2} \text {, Victorian perinatal data collection }{ }^{3} \text {, WA perinatal data collection }{ }^{4} \text {, } \\
\text { NSW perinatal data collection }\end{array}$} \\
\hline
\end{tabular}


Table 151 shows a comparison of measures of accuracy and reliability for common conditions reported to both the ISC and other state perinatal data collections, where published information is available. On the limited information available, reporting of pregnancy related conditions to state perinatal data collections has similar characteristics to the ISC: high levels of specificity, high NPVs, generally high levels of overall agreement, and a wide variation in sensitivities. Compared with perinatal data collections the accuracy of reporting to the ISC was better for pre-existing hypertension, worse for premature rupture of membranes, pre-eclampsia and postpartum haemorrhage, and similar for other conditions for which comparative information was available.

\section{Discussion}

Hospital administrative data have the advantage of being routinely collected and are therefore inexpensive as a resource for monitoring perinatal health. However, there has been reluctance on the part of clinicians to use such data due to concerns about data quality. This review provides evidence of the accuracy of ISC data based on a review of large random sample of mother and baby records. Not all conditions that might be of interest to clinicians occurred in our sample. However, results for common conditions of clinical interest are presented, and information on patterns of accuracy of less common conditions helps provide a picture of the value of the ISC as a source of information on perinatal health.

We found that the accuracy and reliability of the ISC is characterised by:

- variable levels of overall agreement, with about half of all conditions reported at a level considered to be excellent agreement beyond chance and a further one third at a level indicating fair to good agreement beyond chance;

- high levels of specificity, indicating that false positive reports are uncommon;

- variable levels of sensitivity, indicating a variable level of under-enumeration;

- generally higher levels of accuracy and reliability for neonatal records than maternal records.

The findings of overseas studies are similar in terms of variable levels of sensitivity and high levels of specificity and in both the perinatal area, ${ }^{11,12}$ and for morbidities generally..$^{13}$ A population based Canadian study found that the prevalence of a range of pregnancy conditions reported from hospital admission records was within a reasonable range of those reported in the literature.${ }^{14}$ From the limited amount of published information available, it appears that state perinatal data collections have similar characteristics, although we could only make comparisons for a small number of maternal conditions and some of the published studies are several years old and may not reflect current levels of accuracy.
This review assessed the extent to which the ISC records conditions documented in the medical record. The Australian Coding Standards permit clinical coders to code a condition only if the diagnosis is documented in the medical record or otherwise confirmed by a clinician. For example, the Coding Standards state that postpartum haemorrhage (PPH) is a haemorrhage of $500 \mathrm{mls}$ or more in the case of a vaginal delivery and $750 \mathrm{mls}$ or more in the case of a caesarean section. However, if the diagnosis of PPH is not documented in the medical record, it should be confirmed by a clinician before it is coded. Thus clinical coders are not permitted to infer a diagnosis. Confirmation of a suspected diagnosis is unlikely to occur in a busy medical record department and, in practice, it is likely that a condition will generally only be coded if the diagnosis is clearly documented in the medical record. The higher levels of accuracy found for neonatal versus maternal conditions in this study may reflect better documentation of diagnoses for babies than mothers. Levels of documentation may also by influenced by screening practices, for example, for gestational diabetes.

Further, the Coding Standards state that, in general, a condition may be coded if it affects patient management. Thus, essential hypertension will not be coded if it did not affect the management of the mother during her hospital stay. In addition, hospitals may sometimes be directed by state health authorities or their own administrators to vary their coding practice from the national standard for local reasons, thus introducing inconsistent practices between states and territories, and between hospitals.

These results have several implications for use of hospital data for the purpose of monitoring patterns in the health of mothers and babies:

1. The less than perfect sensitivities and high specificities found in this review suggest that ISC data could be used to measure the burden of disease in mothers or infants where the condition is common and there is independent evidence that enumeration of cases is reasonable. Our results suggest that ISC data could be used to assess the number of cases of diagnosed gestational diabetes, placenta praevia, prolonged pregnancy, vaginal laceration, and possibly pre-existing hypertension.

2. For rare conditions, even a high specificity can result in a substantial proportion of cases reported to the ISC being false positives. For example, maternal care for isoimmunisation has a specificity of 99.8 per cent. However, of the 7 cases reported to the ISC, 5 were true positives ( $7 \mathrm{XPPV}=7 \mathrm{X} 71.4$ per cent $=5$ ) and 2 were false positives. In a hospital setting, it would be advisable to confirm reporting of rare conditions by reference to the medical record.

3. Even where under-enumeration exists, if the condition is common, the specificity is high (that is, there are few false positives), and there is evidence that the level of under-enumeration has not changed over time, then 
the data could be used to indicate whether the burden of disease is increasing or decreasing over time.

4. For conditions with high PPVs and NPVs, ISC data could be used as a source of information for certain study types, such as multivariate analyses of factors (for example demographic factors and hospital insurance status) thought to be associated with the condition, or as a sampling frame for nested case-control studies.

5. Conditions examined in this review are more likely to be studied as risk factors for birth outcomes rather than as outcomes themselves. If the degree of misclassification of mothers and babies to not having a condition when they have it, or vice versa, is not dependent on the birth outcome, then this is known as non-differential bias and may result in a reduction in the estimate of risk found. Alternatively, the ascertained risk could be viewed as an estimated minimum level of risk, which is nevertheless valuable information. There are no published validation studies that have been carried out using this approach in the area of perinatal health. A Canadian validation study found that multivariate risk models for all-cause mortality from percutaneous coronary intervention that were constructed from a provincial hospitalisation database were almost identical to the same models constructed from data obtained through medical record review, despite a variable level of misclassification (particularly under-enumeration) of comorbidities on the hospital database. ${ }^{15}$ The nature and degree of likely bias should be considered separately for individual studies.

6. Recognising the strengths and limitations of hospital administrative data, it has been proposed that such data are useful as a screening tool for identifying problems in quality of health care. ${ }^{16,17}$

Thus, while hospital administrative data such as the NSW ISC have the advantage of being readily available and inexpensive, data quality is a valid concern. The quality of hospital administrative data could be improved by clinicians recognising that clinical coders are not permitted to make diagnoses and ensuring that relevant diagnoses are documented in the medical record; and through improvements in the quality and consistency of coding practice. If the quality of hospital administrative data is viewed critically in terms of the purpose of its use, the data can be a useful resource for monitoring the health of mothers and babies.

\section{Acknowledgements}

This project was funded partially by HERON, a collaborative program auspiced by the Sax, the University of New South Wales, University of Technology Sydney, The Cancer Council NSW and, the NSW Department of Health. HERON is funded by an NHMRC Capacity Building Grant in Population Health Research. We wish to thank participating hospitals for their support and staff of medical records departments for their assistance. Dr Annabelle Chan, Ms Vivien Gee and Ms Kerry Haynes provided assistance in identifying published reports of validation studies carried out on interstate perinatal data. Dr Christine Roberts and Mr Gaston Arnolda of the Centre for Perinatal Health Services Research provided critical comments on the draft paper.

\section{References}

1. Haynes K, Stone C, King J. Major conditions associated with childbirth in Australia: Obstetric haemorrhage and associated hysterectomy. Melbourne: Department of Human Services, 2004.

2. McLean A, Scott J, Keane RK, Sage L, Chan A. Validation of the 1994 South Australian Perinatal Data Collection Form. Adelaide: Department of Human Services, 2001.

3. Vagg L, Taylor O, Riley M, Palma S, Halliday J. Validation of the Victorian Perinatal Morbidity Statistics form: new items, pre-coded text and free text. Health Inf Manag.1999-2000; 29: 118-22.

4. Gee V, Dawes V. Validation Study of the Western Australian Midwives' Notification System 1992. Perth: Health Department of Western Australia, 1994.

5. Taylor L, Pym M, Bajuk B, Sutton L, Travis S, Banks C. New South Wales Mothers and Babies 1998. Sydney: NSW Department of Health, 2000.

6. Riley M, Halliday J. The accuracy of eclampsia cases reported to the Victorian Inpatient Minimum Database and the Perinatal Data Collection Unit. Health Inf Manag. 1998; 28: $13-15$.

7. The International Statistical Classification of Diseases and Related Health Problems, 10th Revision, Australian Modification (ICD-10-AM). Sydney: National Centre for Classification in Health, 1998.

8. The SAS System for Windows version 8.02. Cary, NC: SAS Institute, 1996.

9. Fleiss J. Statistical Methods for Rates and Proportions. New York: John Wiley and Sons, 1981.

10. National Health and Medical Research Council. When does quality assurance in health care require independent ethical review? Canberra: NHMRC, 2003.

11. Korst LM, Gregory KD, Gornbein JA. Elective primary caesarean delivery: Accuracy of administrative data. Paediatr Perinat Epidemiol. 2004; 18: 112-119.

12. Parrish KM, Holt VL, Connell FA, Williams B, LoGerfo JP. Variations in the accuracy of obstetric procedures and diagnoses on birth records in Washington State, 1989. Am J Epidemiol. 1993; 138: 119-27.

13. Quan H, Parsons GA, Ghali WA. Validity of information on comorbidity derived from ICD-9-CM administrative data. Med Care. 2002; 40: 675-85.

14. Wen SW, Liu S, Marcoux S, Marcoux S, Fowler D. Uses and Limitations of Routine Hospital Admission-Separation Records for Perinatal Surveillance. Chronic Dis Can 1997; 18: 113-9.

15. Humphries KH, Rankin JM, Carere RG, Buller CE, Kiely FM, Spinelli JJ. Co-morbidity data in outcomes research: are clinical data derived from administrative databases a reliable alternative to chart review?. J Clin Epidemiol 2000;53:343-9.

16. Schwartz RM, Gagnon DE, Muri JH, Zhao QR, Kellogg R. Administrative data for quality improvement. Pediatrics. 103:291-301.

17. Iezzoni LI. Assessing quality using administrative data. Ann Intern Med. 1997; 127:666-74. 